Please quote as: Blohm, I.; Leimeister, J. M.; Rieger, M. \& Krcmar, H. (2011): Controlling von Ideencommunities - Entwicklung und Test einer IdeencommunityScorecard. In: Controlling, Ausgabe/Number: 2, Vol. 23, Erscheinungsjahr/Year: 2011. Seiten/Pages: 96-103. 


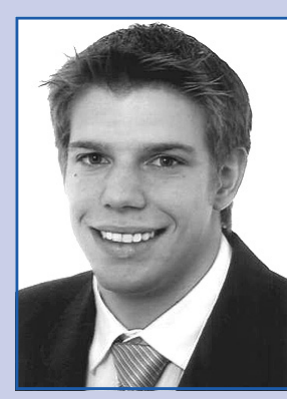

Dipl.-Kfm. Ivo Blohm ist wissenschaftlicher Mitarbeiter am Lehrstuhl für Wirtschaftsinformatik (I17) der Technischen Universität München.

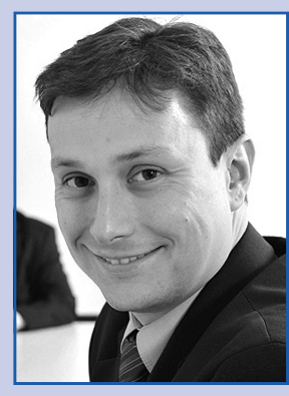

Prof. Dr. Jan Marco Leimeister ist Lehrstuhlinhaber für Wirtschaftsinformatik der Universität Kassel.

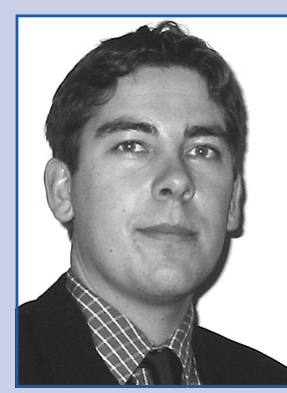

Dipl.-Inf. Markus Rieger ist Geschäftsführer der HYVE Innovation Community AG, München.

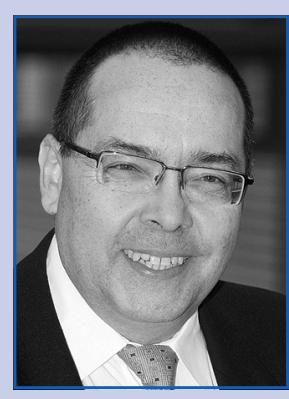

Prof. Dr. Helmut Krcmar ist Lehrstuhlinhaber für Wirtschaftsinformatik (I17) der Technischen Universität München.

Stichwörter

- Balanced Scorecard

- Ideencommunity

- Innovationscontrolling

- Open Innovation

- Virtuelle Community

\section{Controlling von Ideencommunities - Entwicklung und Test einer Ideencommunity-Scorecard}

Viele Unternehmen öffnen ihren Innovationsprozess zunehmend für äußere Einflüsse. Insbesondere der Integration der eigenen Kunden wohnt dabei ein hohes Potential für die Entwicklung neuer Produkte und Dienstleistungen inne. Internetbasierte, unternehmensinitiierte Ideencommunities, in denen Kunden Verbesserungsvorschläge und Innovationsideen einreichen und weiterentwickeln können, ermöglichen eine effiziente Kundeneinbindung. Erfolgreiche Ideencommunities entwickeln sich äußerst dynamisch und entziehen sich klassischen Controlling-Verfahren. In diesem Artikel wird aufbauend auf Ansätzen des Innovationscontrollings und des Controllings von Virtuellen Communities, eine IdeencommunityScorecard entwickelt und am Beispiel der SAPiens-Ideencommunity getestet. Der erarbeitete Ansatz hat sich dabei als sehr erfolgsversprechendes Instrument zum Ideencommunity-Controlling herausgestellt.

\section{Ideencommunities und Controlling}

Seit Beginn des 21. Jahrhunderts kommt es in der Innovationsentwicklung zunehmend zu einem Paradigmenwechsel. Neue Produkte werden nicht mehr allein in geschlossenen Innovationsprozessen entwickelt, sondern eine Öffnung des Innovationsprozesses für externe Einflüsse gewinnt zunehmend an Bedeutung. Kunden sind heute nicht mehr nur noch passive Empfänger und Konsumenten in einer von Herstellern dominierten Wertschöpfung. Sie gestalten vielmehr Produkte und Dienstleistungen aktiv mit und übernehmen dabei sogar teilweise Entwicklung und Herstellung (Reichwald/Piller, 2009). Unternehmenszentrierte Ideencommunities als Ansatz der Kundenintegration erfreuen sich in der Praxis einer steigenden Beliebtheit (Bretschneider, 2010). Erfolgsbeispiele sind Dell IdeaStorm oder My Starbucks Idea. Diese Communities veranschaulichen mit jeweils weit über 10.000 Ideen das enorme Potential dieses Ansatzes für die Produktentwicklung und verdeutlichen zugleich viele weitere Möglichkeiten für andere Anspruchsgruppen. Solche Communities spielen heute eine wesentliche Rolle im Kundenbeziehungsmanagement, für die Selbstdarstellung des Unternehmens und auch für das Recruiting neuer Mitarbeiter.
Um das volle Potential dieser virtuellen Innovationsgemeinschaften ausnutzen $\mathrm{zu}$ können, bedarf es eines ganzheitlichen und auf die Unternehmensziele ausgerichteten Controllinginstruments. Traditionelle Ansätze der Kosten- und Leistungsrechnung stoßen hier an ihre Grenzen und geeignete Verfahren für ein Controlling von Open Innovation Ansätzen fehlen (Reichwald/Piller, 2009). Hilgers/ Piller (2009) haben mit einer Open Innovation Scorecard einen ersten Ansatz zum Controlling von offenen Innovationsprozessen entwickelt, jedoch ist dieser in Bezug auf Ideencommunities in der Praxis nur schwer anwendbar. Viele der von ihnen vorgeschlagenen Kennzahlen lassen sich für Ideencommunities nur unter einem hohen Aufwand und mit einem langen Zeithorizont erheben. Durch die ausschließliche Fokussierung auf die Innovationsentwicklung, werden zudem wesentliche Zielgrößen von Ideencommunities, wie z. B. deren Nutzung als Marketinginstrument, nicht beachtet.

Diese Lücke soll durch diesen Beitrag geschlossen werden, indem wir eine Balanced Scorecard für das Controlling von unternehmenszentrierten Ideencommunities vorstellen und hinsichtlich Umsetzbarkeit testen. Dafür werden auf Basis des Community Building \& Community Management Modells von Leimeister/ Krcmar (2006), Erfolgs- und Steuerungs- 
größen für Ideencommunities abgeleitet und zu einer Ideencommunity-Scorecard verdichtet. Anschließend werden die Anwendbarkeit und die Nützlichkeit des erarbeiteten Konzepts am Beispiel der SAPiens-Ideencommunity unter Beweis gestellt sowie Umsetzungsempfehlungen für die Praxis abgeleitet.

\section{Theoretische Grundlagen}

\section{Ideencommunities und Open Innovation}

Open Innovation ist eine Abkehr vom klassischen Innovationsprozess, der sich weitgehend innerhalb der eignen Unternehmensgrenzen abspielt und in dem ausschließlich Ideen aus unternehmensinternen Bereichen, insbesondere der Forschung und Entwicklung, kommerzialisiert werden (Reichwald/Piller, 2009). In offenen Innovationsprozessen bringen Unternehmen sowohl eigene als auch unternehmensfremde Ideen zur Marktreife bzw. entwickeln Wege, Innovationen auch außerhalb der Unternehmensgrenzen, bspw. in strategischen Allianzen, zu einem Erfolg zu führen (Chesbrough, 2003). Dieser vielschichtige und offene Such- und Lösungsprozess wird in Abb. 1 dargestellt.

Eines der dem Open Innovation Ansatz zugrundeliegenden Prinzipien ist das Nutzbarmachen der sog. "Weisheit der
Vielen“. Dieses Prinzip der kollektiven Intelligenz besagt, dass innerhalb einer sozialen Gemeinschaft durch den Beitrag jedes Einzelnen bessere Entscheidungen erzeugt werden können als durch einzelne Experten oder Individuen. Ideencommunities knüpfen genau an diesem Punkt an. Nach Bretschneider (2010) sind sie ein von Unternehmen eingesetztes Instrument zur Integration von Kunden oder Produktanwendern in die frühen Phasen des Innovationsentwicklungsprozesses. Diese Virtuellen Communities (VC) werden direkt von Unternehmen initiiert und betrieben. Kunden und Produktanwender können auf einer Internetplattform Innovationsideen aus dem Produkt- oder Unternehmensumfeld einreichen und mit anderen Ideengebern in Bezug auf eingestellte Ideen kollaborieren, bspw. durch Ideenkommentierung, -bewertung und/oder -weiterentwicklung. Der dabei forcierte CommunityCharakter soll die Kreativität und Qualität der Beiträge durch das Zusammenwirken verschiedener Kunden bzw. Produktanwender mit unterschiedlichen Wissen, Stärken und Erfahrungen anregen.

\section{Controlling von virtuellen Communities}

Das Community-Building und Community-Management-Modell (Leimeister) Krcmar, 2006) veranschaulicht wie VCs durch Unternehmen systematisch aufgebaut, betrieben und entwickelt werden können (vgl. Abb. 2). Das CommunityControlling nimmt dabei eine zentrale Rolle ein und ermöglicht, den Erfolg einzelner Community-Angebote abzuschätzen, bei Zielabweichungen Maßnahmen zu ergreifen und die Wirksamkeit der getroffenen Maßnahmen zu überprüfen. Es umfasst die Definition von geeigneten Evaluationsmethoden und -kriterien. Die erforderlichen Daten werden direkt aus der Community gewonnen. Mittels quantitativer Logfile-Analysen kann das Verhalten der Mitglieder, z. B. die Verweildauer auf den einzelnen Communityseiten, analysiert werden. Aber auch die inhaltliche Analyse der Nutzungsartefakte, wie z. B. der Mitgliederkommentare, oder kontinuierliche Mitgliederbefragungen sind wichtige Instrumente zur Identifikation von Problemen und Verbesserungspotentialen (Leimeister/Krcmar, 2006; Stieglitz, 2008).

Der Erfolg einer VC kann aus Sicht der Mitglieder und aus Sicht des Betreibers gemessen und gesteuert werden. So kann nur eine für die Mitglieder gut funktionierende und attraktive VC erfolgreich für den Betreiber erfolgreich sein (z. B. im Sinne von Kundenbindung). Preece (2001) identifiziert u. a. Anzahl der Nachrichten und Mitglieder, Nachrichten pro Mitglied, Art und Anzahl von Aus-

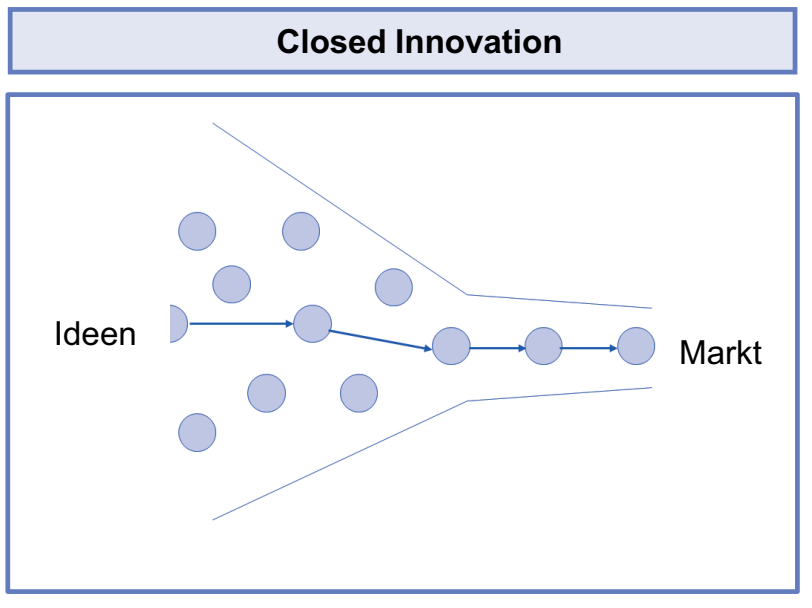

Entwicklung von Ideen und deren Kommerzialisierung erfolgt ausschließlich über unternehmensinterne Ressourcen

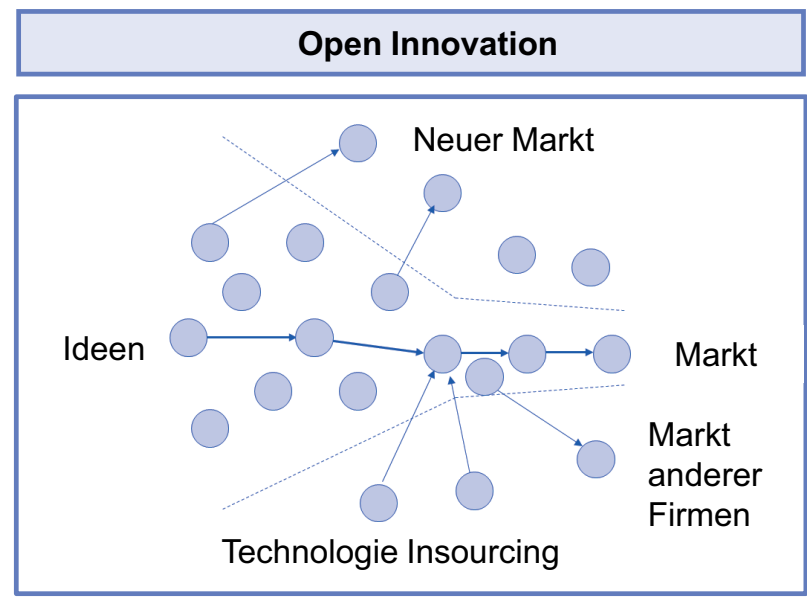

Entwicklung von Ideen und deren Kommerzialisierung erfolgt auch über unternehmensexterne Ressourcen, z.B. via Ein-/ Auslizensierung von Patenten, Kooperationen und Spin Outs

Abb. 1: Open und Closed Innovation (in Anlehnung an Chesbrough (2003)) 


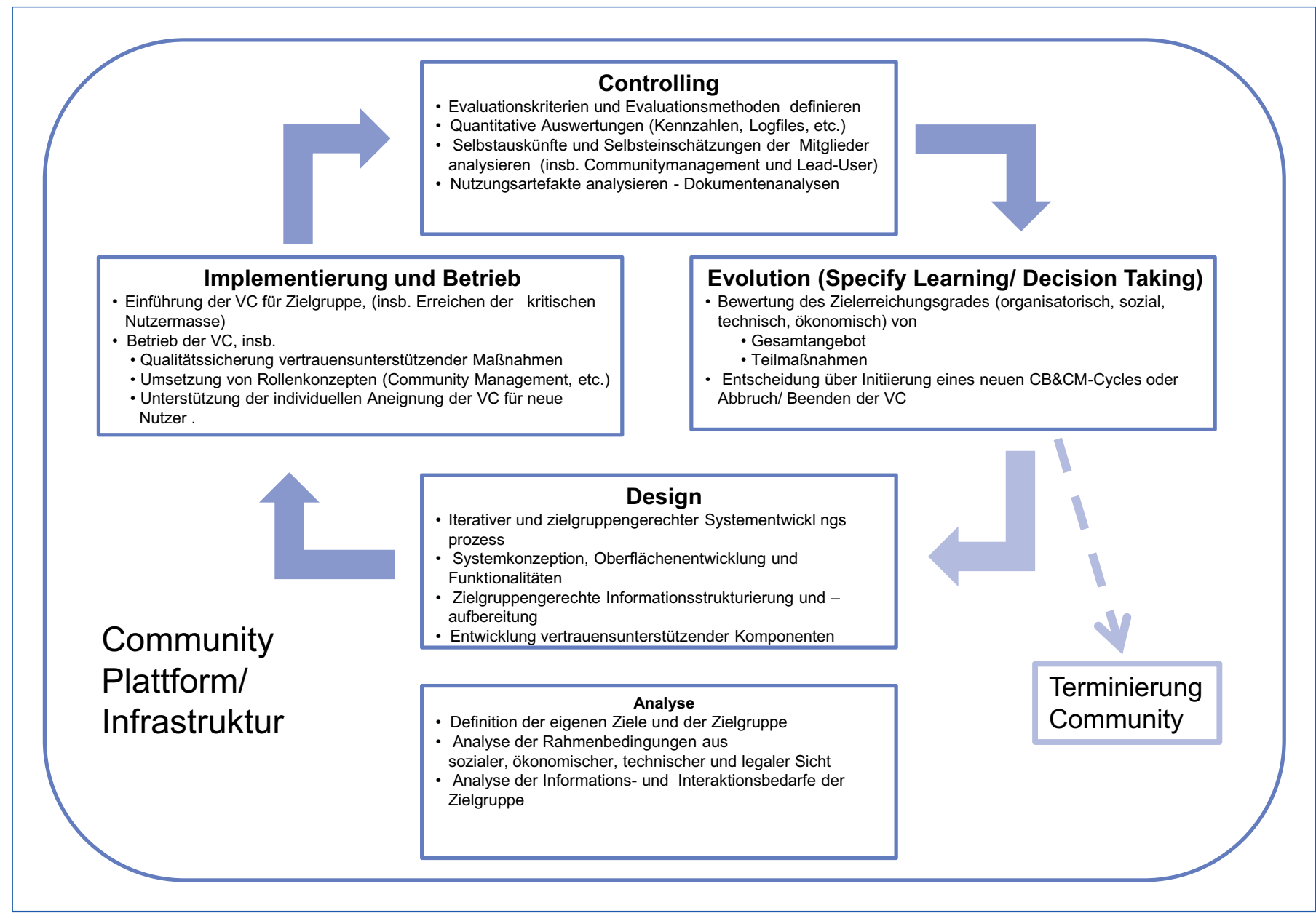

Abb. 2: Community-Building und Community-Management Modell (Leimeister/Krcmar (2006))

tauschverhältnissen und Qualität von Beiträgen als Erfolgsfaktoren aus Sicht der Mitglieder, welche die für den Betreiber relevanten Erfolgsgrößen Seitenzugriffe, Verweildauer und Besuchshäufigkeit beeinflussen. Weitere Erfolgsgrößen für den Betreiber orientieren sich oftmals an Zielgrößen des Marketings und können u. a. Imageverbesserung, Kundenbindung oder Gewinne in Form von zusätzlichen Verkäufen oder Werbeeinahmen darstellen (Cothrel, 2000).

\section{Innovationscontrolling}

Brockhoff (1999) definiert Innovationscontrolling als die ergebnisorientierte Fokussierung auf sämtliche Prozesse (Planung, Kontrolle und Evaluation), welche darauf zielen neues technisches Wissen im Hinblick auf Produkte, Prozesse und Anwendungen zu generieren. Dabei ist grundsätzlich zwischen Forschung und Entwicklung $\mathrm{zu}$ differenzieren. Forschung beschäftigt sich mit der Gewinnung neuer Erkenntnisse, die in der Entwicklung in neue Produkte überführt werden (vgl. Abb. 3). Je weiter man im Innovationsprozess voranschreitet, also

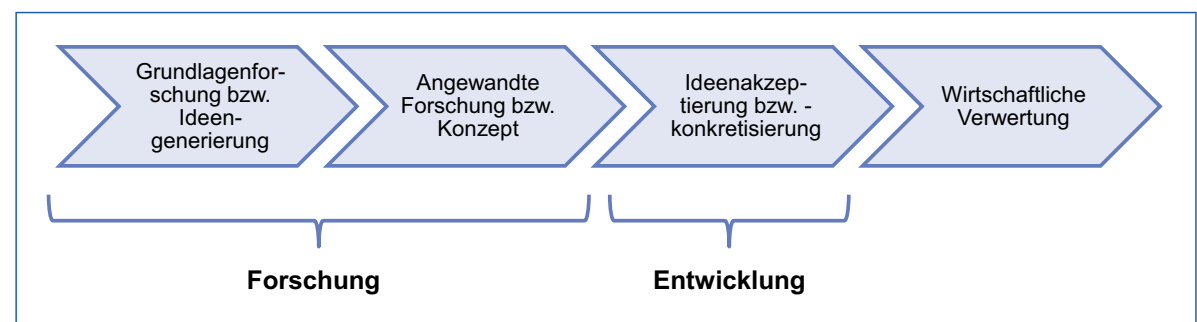

Abb. 3: Begriffsabgrenzungen im Innovationsprozess (in Anlehnung an Faber, 2008)) vom Bereich der Forschung in den der Entwicklung kommt, desto eher werden klassische Controllingverfahren anwendbar. Im Bereich der Entwicklung sind die Prozesse meist gut strukturiert und deren Ergebnisse einfach erfassbar. Bei der Forschung ist eine Erfolgskontrolle und -steuerung ungleich schwieriger. Aufgrund hoch variabler und unstrukturierter Prozesse auf neuen Gebieten, lassen sich Ergebnisse am "fuzzy front end“ häufig nur schwer messen und bewerten. Startpunkte für ein Performance Measurement können hier jedoch z. B. Personalkosten, Anzahl und Qualität der Ideen und Erfahrung der Mitarbeiter darstellen (Janssen/Möller, 2009).

\section{Entwicklung einer Ideencommunity-Scorecard}

Für viele Betreiber ist das Aufbauen einer Ideencommunity in der Regel selbst eine Innovation. Daraus ergeben sich für diesen ein hohes Maß an Unsicherheit, eine 
hohe Variabilität und eine geringe Strukturierung der Prozesse zum Management und Einbindung der Community. Viele Erfolgsgrößen, wie z. B. die Qualität der eingereichten Ideen, sind nur schwer quantifizierbar und die Ziele unterschiedlicher Anspruchsgruppen müssen beim Aufbau berücksichtigt werden. Für ein Controlling von Ideencommunities greifen daher sowohl Ansätze des Controllings von VC als auch des Innovationscontrollings zu kurz (vgl. Abb. 4). Während erstere keinen Bezug zur Innovationsentwicklung besitzen, sind letztere in Bezug auf Ideencommunities nur in einem sehr begrenzten Umfang anwendbar. Kennzahlen für ein Performance Measurement von Produktinnovationen oder Open Innovation sind in Ideencommunities in der Praxis nicht oder nur langristig erhebbar. Weiterhin werden durch diese rein auf die Innovationsentwicklung ausgerichteten Controlling-Ansätze wesentliche Zielgrößen von Ideencommunities außen vor gelassen.

Aufbauend auf dem Ansatz von Hilgers/ Piller (2009) wird im folgenden eine Ideencommunity-Scorcard entwickelt. Eine Balanced Scorecard (BSC) ist eine Systematik zur ganzheitlichen Steuerung der Unternehmensstrategie, mit der die aus der Vision des Unternehmens abgeleitete Strategie durch vier verschiedene Managementperspektiven operationalisiert wird. Für die vier Perspektiven Finanzen, Kunden, Innovationsprozess sowie Lernen/externe Akteure werden auf jeweils einer Scorecard Untersuchungsobjekte und deren Kennzahlen festgelegt, Ziele für diese Objekte definiert sowie Initiativen zur Erreichung der Ziele festgehalten. Die Ideencommunity-Scorecard ist in Abb. 5 einsehbar.

\section{Innovationsprozess}

In Bezug auf die Entwicklung neuer Produkte stellen Quantität und Qualität der eingereichten Ideen zentrale Zielgrößen einer Ideencommunity dar. Kundenideen sind kreative Produkte, die häufig nur einen geringen Reifegrad und eine niedrige Spezifität besitzen (Blohm et al., 2010). Für eine kurzfristige Quantifizierung deren Nutzens muss daher auf Verfahren der Kreativitätsforschung, wie z. B. die Consensual Assessment Technique (Amabile, 1996), zurückgegriffen werden, das zur Bewertung von Kundenideen gut geeignet ist (Blohm et al.,

\begin{tabular}{|c|c|c|}
\hline Controlling-Objekt & Virtuelle Community & Forschung und Entwicklung \\
\hline Oberste Ziele & $\begin{array}{l}\text { Kundenbindung } \\
\text { Verkaufssteigerung }\end{array}$ & $\begin{array}{l}\text { - Generierung von Innovationen } \\
\text { - Wissenserwerb }\end{array}$ \\
\hline $\begin{array}{l}\text { Zentrale Control- } \\
\text { ling-Instrumente }\end{array}$ & $\begin{array}{l}\text { - Logfile-Analyse } \\
\text { - Mitglieder-Befragung }\end{array}$ & $\begin{array}{l}\text { Kennzahlensysteme } \\
\text { - Budgetierung } \\
\text { - Prozesskostenrechnung } \\
\text { - Projestitionsrechnung } \\
\text { - Projelplanung und -kontrolle }\end{array}$ \\
\hline $\begin{array}{l}\text { Schwachstellen } \\
\text { in Bezug auf } \\
\text { Ideencommunity- } \\
\text { Controlling }\end{array}$ & $\begin{array}{l}\text { kein Innovationsbezug } \\
\text { - Uneinheitliche Erfolgs- } \\
\text { faktoren und Kennzah- } \\
\text { len } \\
\text { - Wirkzusammenhänge } \\
\text { wenig erforscht } \\
\text { - Wenig systematische } \\
\text { Verfahren }\end{array}$ & $\begin{array}{l}\text { Kennzahlen für Ideencommunities } \\
\text { nicht/nur langfristig erhebbar } \\
\text { Dynamik von Ideencommunities } \\
\text { nicht abbildbar } \\
\text { Schlechte Bewertbarkeit von Er- } \\
\text { folgsgrößen (Ideenqualität) } \\
\text { Mangelnde Berücksichtigung von } \\
\text { Zielen aus Marketing und Personal }\end{array}$ \\
\hline
\end{tabular}

Abb. 4: Community- und Innovationscontrolling

2019). In Bezug auf die Ideenqualität ist der Anteil guter Ideen, die ein a priori vom Betreiber definiertes Mindestqualitätsmaß überschreiten, an allen Ideen von Bedeutung, da letztendlich nur diese das innovationsrelevante Ergebnis einer Ideencommunity darstellen. Schlechte, bereits bekannte oder mittelmäßige Ideen sind für den Betreiber im Prinzip gleichermaßen wertlos, da sie nicht weiter verfolgt werden. Werden Ideen für eine unternehmensinterne Weiterentwicklung aufgegriffen, entstehen aus ihnen in der Regel Folgeprojekte, die Umsetzung der Idee in ein konkretes Produkt prüfen.

Neben der Möglichkeit neue Ideen einzureichen, haben Teilnehmer auch die Möglichkeit, Ideen anderer zu bewerten und mittels Kommentar- oder WikiFunktionen zu überarbeiten. Dabei stellen die Community-Bewertung an sich als auch die Anzahl der Community-Bewertungen pro Idee wesentliche Indikatoren für die von den Community-Mitgliedern wahrgenommene Ideenqualität als auch für deren Verallgemeinerbarkeit auf andere Kundensegmente dar. Die Anzahl der Kommentare bzw. die Überarbeitungen pro Idee können weiteren Aufschluss über die Beliebtheit und aber auch über die Reife einer Idee liefern. Je öfter einer Idee durch die Mitglieder überarbeitet wurde, desto größer ist in der Regel auch deren Qualität (Blohm et al., 2010).

\section{Kunden}

Neben der Innovationsperspektive ist insb. die kundenbezogene Sichtweise ausschlaggebend für den Erfolg einer Ideencommunity. Wesentliche Zielgrößen aus dem Controlling von VC stellen Aktivität und Reichweite dar. Während die Aktivität die Anzahl der durch die Teilnehmer vollzogenen Aktionen wie z. B. die Kommunikationsfrequenz umfasst (Hilgers/ Piller, 2009), beschreibt die Reichweite z. B. die Größe der Community oder die Anzahl der einzelnen Websiteaufrufe ( $\mathrm{Pa}$ ge Impressions) der Ideencommunity. Einfache, aber aussagekräftige Aktivitätsmaße stellen die Anzahl der Logins pro User, die Ideengeberquote (Anteil von Ideengebern an allen Teilnehmern) oder die Ideenquote (Verhältnis von Ideen zu Teilnehmern) dar, die sich direkt aus den Logdaten generieren lassen. Von Bedeutung ist weiterhin die Anzahl an sog. „Super Usern“. In nahezu allen VCs gibt es eine kleine Anzahl von besonders aktiven Teilnehmern, die für einen Großteil, der in Community produzierten Inhalte verantwortlich sind und für Aufbau und Betrieb der Ideencommunity von zentraler Bedeutung sind (Daum, 2007). Neben eher quantitativen Kennzahlen wie Größe und Reichweite einer Ideencommunity sind aus Kundenperspektive auch qualitative Faktoren zu betrachten (Stieglitz, 2008). Aus Kundenperspektive sind hier für Ideencommunities insb. die Identifikation mit dem Betreiber und deren Involvement von Interesse. Die Charakteristika und Fähigkeiten der einzelnen Mitglieder sind weitere Beobachtungsgrößen (Hilgers/Piller, 2009), da Ideencommunities in einen hohe Maße sog. Lead User anziehen - Kunden, die selbst innovativ fähig werden und deren Bedürfnisse mit der Zeit für den Massenmarkt von Bedeutung werden (Reichwald/Piller, 2009). 


\begin{tabular}{|c|c|c|c|c|}
\hline \multicolumn{2}{|r|}{$\begin{array}{l}\text { Kunden } \\
\text { Kennzahlen }\end{array}$} & & \multicolumn{2}{|r|}{$\begin{array}{l}\text { Innovationsprozess } \\
\text { Kennzahlen }\end{array}$} \\
\hline \multirow[t]{2}{*}{$\begin{array}{l}\text { Aktivität und } \\
\text { Reichweite }\end{array}$} & \multirow{2}{*}{$\begin{array}{l}\text { Ideenquote / Ideengeberquote } \\
\text { Logins / Logins pro Mitglied } \\
\text { Page Impression / PI pro Mitglied }\end{array}$} & & $\begin{array}{l}\text { Ideen- } \\
\text { quantität }\end{array}$ & Ideen \\
\hline & & & Ideen- & Gute Ideen / Anteil guter Ideen \\
\hline \multirow{2}{*}{$\begin{array}{l}\text { Größe und } \\
\text { Zusammen- } \\
\text { setzung }\end{array}$} & \multirow{2}{*}{$\begin{array}{l}\text { Mitglieder } \\
\text { Super User } \\
\text { Lead Userness der Mitglieder } \\
\text { Produktwissen der Mitglieder }\end{array}$} & & qualital & $\begin{array}{l}\text { Überarbeitungen pro Idee } \\
\text { Zeichen pro Idee }\end{array}$ \\
\hline & & & $\begin{array}{l}\text { Ideen- } \\
\text { bewertung }\end{array}$ & $\begin{array}{l}\text { Communitybewertung } \\
\text { Bewertungen pro Idee }\end{array}$ \\
\hline $\begin{array}{l}\text { Kunden- } \\
\text { bindung }\end{array}$ & $\begin{array}{l}\text { Identifkation der Mitglieder } \\
\text { Involvement der Mitglieder }\end{array}$ & & $\begin{array}{l}\text { Ideen- } \\
\text { realisierung }\end{array}$ & $\begin{array}{l}\text { Folgeprojekte } \\
\text { Umgesetzte Ideen }\end{array}$ \\
\hline & & $\begin{array}{l}\text { Vision } \\
\text { und } \\
\text { Strategie }\end{array}$ & & \\
\hline \multicolumn{2}{|c|}{ Lernen / externe Akteure } & & \multicolumn{2}{|c|}{$\begin{array}{ll} & \text { Finanzen } \\
\text { Zielgröße } & \text { Kennzahlen } \\
\end{array}$} \\
\hline $\begin{array}{l}\text { Lernen } \\
\text { Community }\end{array}$ & $\begin{array}{l}\text { Identifikation neuer Trends } \\
\text { Identifikation neuer Bedürfnisse } \\
\text { Registrierte Mitarbeiter } \\
\text { Mitarbeiter Logins } \\
\text { Mitarbeiter Page Impressions }\end{array}$ & & Kosten & $\begin{array}{l}\text { IT-Plattform } \\
\text { Community-Management Marketing } \\
\text { Preise } \\
\text { Ideenbewertung } \\
\text { Mitarbeiter }\end{array}$ \\
\hline $\begin{array}{l}\text { Neue } \\
\text { Mitarbeiter }\end{array}$ & $\begin{array}{l}\text { Attraktivität als Arbeitgeber } \\
\text { Praktikanten } \\
\text { Feste Mitarbeiter }\end{array}$ & & Erlöse & $\begin{array}{l}\text { Einnahmen aus umgesetzen Ideen } \\
\text { Kosteneinsparungen aus umgesetzen } \\
\text { Ideen }\end{array}$ \\
\hline
\end{tabular}

Abb. 5: Ideencommunity-Scorecard

\section{Lernen und externe Akteure}

Nach Cohen/Levinthal (1990) ist Lernen eine der wesentlichen Aufgaben von Forschung und Entwicklung, da diese eine wichtige Quelle für die Wissensbasis von Unternehmen darstellt. In den von den Mitgliedern entwickelten Ideen und in der Community geführten Diskussionen lassen sich, z. B. durch inhaltsanalytische Verfahren (Netnography), Rückschlüsse auf die Bedürfnisse der Mitglieder ziehen und durch Verdichtung neue Trends ableiten. Für ein effektives organisationales Lernen und Spill-Over-Effekte auf die Innovationsentwicklung ist die Einbettung von Ideencommunities in den organisationalen Kontext des Betreibers ausschlaggebend. Die Anzahl der in der Ideencommunity registrierten F\&E-Mitarbeiter, sowie deren Logins und Page Impressions stellen hierfür einfach erhebbare Indikatoren dar. Je aktiver sich F\&E-Mitarbeiter in der Community beteiligen, desto umfassender gestaltet sich der Lerntransfer zwischen Ideencommunity und F\&E-Abteilung.
Die Mitglieder von Ideencommunities weisen eine hohe Identifikation mit dem betreibenden Unternehmen und besitzen oftmals ein hohes Produktwissen Träger von für die Innovationsentwicklung wesentlichen Bedürfnis- und Lösungsinformationen (Reichwald/Piller, 2009). Das Rekrutieren neuer Mitarbeiter aus den Mitgliedern einer Ideencommunity ist daher eine weitere wesentliche Zielgröße von Ideencommunities, für das die Anzahl der rekrutierten Praktikanten und festen Mitarbeiter als auch eine allgemeine Einschätzung der Attraktivität des Betreibers als Arbeitgeber wesentliche Indikatoren darstellen.

\section{Finanzen}

Aus einer finanziellen Sicht sind die Kosten und Erträge, die aus einer Ideencommunity resultieren zu betrachten. Wesentlich sind hier vor allem die Kosten für die Erstellung und den Betrieb (Hosting) der IT-Plattform, die Personalkosten für das Community-Management und die Arbeitszeit der Mitarbeiter, die sich an der Ideencommunity beteiligen. Aber auch die Kosten für begleitende Werbemaßnahmen, Preise und andere Anreize für die Community-Teilnehmer sowie für die Bewertung der eingereichten Ideen müssen betrachtet werden. Demgegenüber stehen etwaige Einnahmen bzw. Kosteneinsparungen, die durch die Umsetzung von Kundenideen resultieren (Hilgers/Piller, 2009).

\section{Test der Ideencommunity- Scorecard}

Die entwickelte BSC wurde bei der vom Lehrstuhl für Wirtschaftsinformatik der TU München aufgebauten SAPiens-Ideencommunity des Software-Herstellers $S A P$ einer praktischen Erprobung unterzogen. Ziel der SAPiens-Community ist es $S A P$-Anwendern ein Forum zu schaffen „,neue Ideen rund um $S A P^{“}$ zu entwickeln und auf der IT-gestützten SAPiensPlattform einzureichen. Die Community 
entwickelte sich aus einem Ideenwettbewerb und wurde 2007 ins Leben gerufen. Für die folgende Analyse liegen die Daten des Jahres 2008 zugrunde. Die hier präsentierten Daten wurden durch eine Analyse der Logdaten, eine Befragung des Community-Managers und eine Befragung der Teilnehmer gewonnen. Die im Anhang einsehbaren Items der Teilnehmerbefragung, konnten auf einer RatingSkala von 1 (sehr niedrig) bis 5 (sehr hoch) bewertet werden. In Abb. 6 werden die erhobenen Kennzahlen der SAPiensCommunity zusammenfassend dargestellt.

Im Betrachtungszeitraum wurden 57 Ideen eingereicht, von denen 7 (ca. 12\%) von der Expertenjury als qualitativ hochwertig eingestuft wurden. Jede Idee wurde von den Mitgliedern der Ideencommunity ca. 7 mal bewertet, wobei die durchschnittliche Qualität der Ideen mit einer Bewertung von 1,74 ( $1=$ sehr niedrig; $5=$ sehr hoch) von den Teilnehmer verhalten eingeschätzt wurde. Aus den Ideen ergab sich ein Folgeprojekt, das unternehmensintern weiterverfolgt, aber nicht umgesetzt wurde.

Die 145 Mitglieder loggten sich insgesamt 1428 ein (ca. 10 Logins/Mitglied). Dabei wurden insgesamt 20889 Page Impressions erzielt (ca. 144 Page Impressions/Mitglied). Ca. $27 \%$ aller Teilnehmer reichten $\mathrm{min}$. eine Idee ein und die Ideenquote liegt bei $39 \%$. Unter den Teilnehmern befanden sich zwei besonders aktive Teilnehmer, die ca. $35 \%$ der Ideen einreichten. Die Teilnehmer wiesen eine hohe Identifikation mit SAP (Mittelwert $\mu=3,53)$ und ein hohes Involvement ( $\mu$ $=3,93)$. Produktwissen $(\mu=3,35)$ und Lead Userness $(\mu=3,19)$ der Teilnehmer sind leicht überdurchschnittlich.

Auf Basis der Ideen und Teilnehmerdiskussionen konnten 3 Trends identifiziert werden. Es meldeten sich 13 SAP-Mitarbeiter in der SAPiens-Community an, die sich insgesamt 75 anmeldeten und 129 Page Impressions generierten. Die Attraktivität als Arbeitgeber ist mit einem Mittelwert von 3,64 aus Teilnehmersicht als hoch einzuschätzen. Es wurde ein Mitglied als Praktikant rekrutiert, das anschließend von SAP übernommen wurde.

Beim Aufbau und Betrieb der SAPiensCommunity kann das Community-Management als der Hauptkostentreiber an-

\begin{tabular}{|c|c|c|c|}
\hline Dimension & Zielgröße & Kennzahl & $\begin{array}{l}\text { Wert bei } \\
\text { SAPiens }\end{array}$ \\
\hline \multirow[t]{3}{*}{ Kunden } & Aktivität \& Reichweite & $\begin{array}{l}\text { Ideenquote/Ideengeberquote } \\
\text { Logins/Logins pro Mitglied } \\
\text { Page Impression/PI pro Mitglied }\end{array}$ & $\begin{array}{l}0,39 / 0,27 \\
1428 / 9,85 \\
20889 / 144\end{array}$ \\
\hline & $\begin{array}{l}\text { Größe \& Zusammen- } \\
\text { setzung der IC }\end{array}$ & $\begin{array}{l}\text { Mitglieder } \\
\text { Super User } \\
\text { Lead Userness der Mitglieder } \\
\text { Produktwissen der Mitglieder }\end{array}$ & $\begin{array}{l}145 \\
2 \\
3,19^{*} \\
3,35^{*}\end{array}$ \\
\hline & Kundenbindung & $\begin{array}{l}\text { Identifkation der Mitglieder } \\
\text { Involvement der Mitglieder }\end{array}$ & $\begin{array}{l}3,53^{*} \\
3,93^{*}\end{array}$ \\
\hline \multirow{4}{*}{$\begin{array}{l}\text { Innova- } \\
\text { tions- } \\
\text { prozess }\end{array}$} & Ideenquantität & Ideen & 57 \\
\hline & Ideenqualität & $\begin{array}{l}\text { Gute Ideen/Anteil guter Ideen } \\
\text { Kommentare pro Idee } \\
\text { Überarbeitungen pro Idee } \\
\text { Zeichen pro Idee }\end{array}$ & $\begin{array}{l}7 / 0,12 \\
0,46 \\
0,75 \\
2190\end{array}$ \\
\hline & Ideenbewertung & $\begin{array}{l}\text { Communitybewertung } \\
\text { Bewertungen pro Idee }\end{array}$ & $\begin{array}{l}1,73^{*} \\
6,84\end{array}$ \\
\hline & Ideenrealisierung & $\begin{array}{l}\text { Folgeprojekte } \\
\text { Umgesetzte Ideen }\end{array}$ & $\begin{array}{l}1 \\
0\end{array}$ \\
\hline \multirow[t]{2}{*}{$\begin{array}{l}\text { Lernen/ } \\
\text { Externe } \\
\text { Akteure }\end{array}$} & Lernen & $\begin{array}{l}\text { Neue Trends } \\
\text { Neue Bedürnisstrukturen } \\
\text { Registrierte Mitarbeiter } \\
\text { Mitarbeiter Logins } \\
\text { Mitarbeiter Page Impressions }\end{array}$ & $\begin{array}{l}3 \\
0 \\
13 \\
75 \\
129\end{array}$ \\
\hline & Neue Mitarbeiter & $\begin{array}{l}\text { Attraktivität als Arbeitgeber } \\
\text { Praktikanten } \\
\text { Feste Mitarbeiter }\end{array}$ & $\begin{array}{l}3,64^{*} \\
1 \\
1\end{array}$ \\
\hline \multirow[t]{2}{*}{ Finanzen } & Kosten & $\begin{array}{l}\text { IT-Plattform } \\
\text { Community-Management } \\
\text { Marketing } \\
\text { Preise } \\
\text { Ideenbewertung } \\
\text { Mitarbeiter }\end{array}$ & $\begin{array}{l}- \\
104 \mathrm{PT} \\
0 € \\
10800 € \\
7,5 \mathrm{PT} \\
1,5 \mathrm{PT}\end{array}$ \\
\hline & Erlöse & $\begin{array}{l}\text { Einnahmen aus umgesetzen Ideen } \\
\text { Kosteneinsparungen } \\
\text { aus umgesetzen Ideen }\end{array}$ & $\begin{array}{l}0 € \\
0 €\end{array}$ \\
\hline
\end{tabular}

${ }^{*} \operatorname{Min}=1 ; \operatorname{Max}=5$

Abb. 6: Ideencommunity-Scorecard am Beispiel der SAPiens-Community

gesehen werden, für das durchschnittlich ca. 2 Personentage (PT) pro Woche aufgewandt wurden. Die Expertenbewertung zur Evaluation der eingereichten Ideen belief sich auf etwa 7,5 weitere PT. Für Preise zur Aktivierung und Motivation der Mitglieder wurden insg. 10.800 EUR aufgewendet. Für Marketing-Materialien sind neben der Arbeitszeit des Community-Managers keine Kosten angefallen. Die SAPiens-Community wurde im Rahmen eines Forschungsprojektes aufgebaut - die Kosten für die verwendete IT-Plattform dürfen daher nicht veröffentlicht werden. Da keine der eingereichten Ideen in ein konkretes Produkt überführt wurde oder anderweitig umgesetzt wurde, konnte durch die Ideencommunity keine direkt nachweisbaren Gewinne oder Kosteneinsparungen realisiert werden.

Durch die entwickelte IdeencommunityScorecard konnte der Beitrag der SAPiens-Community für die Innovationsentwicklung und die anderen beteiligten Unternehmensteile sehr gut sichtbar gemacht werden. Weiterhin konnten durch die gewonnenen Kennzahlen die Community weiterentwickelt und Maßnahmen zur gezielten Steuerung der Community abgeleitet werden. Zum Beispiel 
wurden zusätzliche Funktionalitäten zur Ermöglichung von User-Kollaboration implementiert, um die Qualität der generierten Ideen weiter zu steigern.

\section{Umsetzungsempfehlungen}

Balanced Scorecards stellen grundsätzlich immer ein Instrument zur Umsetzung und nicht zur Entwicklung von Strategien dar. Vor der Einführung einer Ideencommunity-BSC ist daher ein gemeinsames Verständnis über die grundsätzlichen Ziele einer Ideencommunity zwischen allen beteiligten Anspruchsgruppen und dem Top-Management zu erzielen. Dabei sind insbesondere der Marketing- und F\&E-Abteilung eines Unternehmens Rechnung zu tragen, da diese oftmals unterschiedliche Ziele mit einer solchen Community verfolgen. Weiterhin ist es für ein effektives Ideencommunity-Controlling notwendig, die Ideencommunity in die übergeordnete Unternehmensstrategie einzubetten und eine Ideencommunity-Strategie zu erarbeiten, damit eine Anpassung der hier vorgeschlagenen BSC an die Ziele des Ideencommuity-Betreibers erfolgen kann.

Weiterhin impliziert die entwickelte Ideencommunity-Scorecard eine weitreichende Verzahnung der Ideencommunity mit dem betreibenden Unternehmen. So wird bspw. angenommen, dass die eingereichten Ideen durch den Betreiber bewertet und die vielversprechendsten Ideen in das interne Innovationssystem eingeschleust werden. Ideencommunities werden in der Praxis jedoch oftmals als „Pilotprojekte“ ins Leben gerufen und als solche nur rudimentär in das Unternehmen eingebunden. Die organisatorische Einbettung in die Prozesse und Strukturen des betreibenden Unternehmens ist aber eine wesentliche Voraussetzung für ein Ideencommunity-Controlling. Zudem ist die Frage zu klären, wie ein Ideencommunity-Controlling organisatorisch ausgestaltet werden soll. Das Community-Management besitzt eine Gatekeeper-Funktion zwischen Unternehmen und Ideencommunity und sollte daher erster Anlaufpunkt sein. Es hat direkten Zugriff auf Mitglieder und Logdaten und kann daher einen Großteil der benötigten Daten direkt beschaffen. Andere Daten, wie z. B. die Expertenbewertung zur Einschätzung der Qualität der Ideen, können nur mit einem hohen Aufwand erhoben werden. Bei der Einführung des Ideencommunity-Controllings ist daher darauf zu achten, dass es nicht zum reinen Selbstzweck wird. Eine Fokussierung auf die für ein Unternehmen und dessen strategische Ausrichtung der Ideencommunity wesentlichen Indikatoren ist daher notwendig, um den Aufwand der Datengewinnung, -analyse und -interpretation handhabbar zu halten. Nur so kann eine langfristige Akzeptanz und Verstetigung des Ideencommunity-Controllings ermöglicht werden.

\section{Zusammenfassung und Ausblick}

Im vorliegenden Artikel wurde eine Ideencommunity-Scorecard entwickelt, deren Anwendbarkeit an einem realen Beispiel unter Beweis gestellt sowie Umsetzungsempfehlungen für die Praxis erarbeitet. Sie ermöglicht ein aus Betreibersicht einfach durchzuführendes Controlling von Ideencommunities, ohne der Community den für die Ideenentwicklung notwendigen Freiraum als „Ideenspielplatz" zu nehmen.

Die ermittelten Kennwerte sind jedoch stark abhängig von der Art der in der Community entwickelten Ideen. Auch die Produktgattung, für die die Ideen generiert wurden, und die adressierbare Zielgruppe von potenziellen Ideengebern spielen in diesem Kontext eine wichtige Rolle. Je nachdem, ob die Teilnehmer text-basierte Ideenbeschreibungen oder graphische Designs entwickeln, werden die Ausprägungen der Kennzahlen stark variieren. Eine Vergleichbarkeit mehrerer Ideencommunities ist somit nur innerhalb eines Typs von Ideen möglich. Weiterhin zeigte das SAPiens-Fallbeispiel, dass kostenbasierte Kennzahlen zum Controlling der SAPiens-Community aus finanzieller Sichtweise einfach erhoben werden können. Jedoch ist eine Gewinnermittlung einzelner umgesetzter Ideen in der Praxis nicht praktikabel und nur unter einem sehr hohen Aufwand durchführbar. Sofern einzelne Kundenideen umgesetzt werden, werden Sie in der Regel nicht in ihrer ursprünglichen Form umgesetzt, sondern werden viel eher unternehmensintern weiterentwickelt, mit anderen Ideen kombiniert oder sie dienen als Anstoß für Neuproduktentwicklungen in einer anderen Produktlinie. Wie unternehmensintern entstandene
Ideen machen die Kundenideen bei der Umsetzung einen Transformationsprozess durch, so dass das Endprodukt nicht mehr viel mit der ursprünglich in der Ideencommunity eingereichten Idee gemein haben muss.

Aus theoretischer Sicht werden durch diesen Artikel zwei wesentliche Beiträge geleistet. Zum einen wird der Ansatz der BSC auf ein neues Anwendungsfeld übertragen. Zum anderen werden Ideencommunities erstmals nicht nur aus Perspektive der Innovationsentwicklung, sondern aus einer ganzheitlichen Sicht betrachtet und damit eine Vielzahl von Anknüpfungspunkten für zukünftige Forschungsaktivitäten aufgezeigt. So ist nicht die Effektivität von Ideencommunities als Instrument des Marketings oder der Personalwesens wenig erforscht, sondern deren Anbindung an unternehmensinterne Prozesse. Weiterhin müssen Methoden und Verfahren entwickelt werden wie neben einer kostenmäßigen auch eine wertorientierte Betrachtung von Ideencommunities aus einer finanziellen Perspektive ermöglichen. Auch besteht Bedarf nach einem Benchmark-Instrument für Ideencommunities, mit dem diese verglichen werden können.

Für die Betreiber von Ideencommunities wurde ein umfassendes Instrument zum Controlling von Ideencommunities entwickelt. Durch dessen kontinuierliche Anwendung kann die dynamische Entwicklung von Ideencommunities abgebildet und deren systematische Steuerung im Hinblick auf die vom Betreiber verfolgten Ziele ermöglicht werden. Die Ideencommunity-Scorecard kann helfen, den Beitrag von Ideencommunities für unterschiedliche Funktionsbereiche wie F\&E, Marketing und Personalwesen darzustellen.

\section{Anhang}

\section{Identifikation}

Ich habe bei SAPiens teilgenommen, weil...

...ich mich mit der Marke SAP identifiziere.

...ich die „SAPiens-Gemeinschaft" gut finde und diese unterstützen möchte.

...ich SAP gut finde und das Unternehmen unterstützen möchte. 


\section{Lead Userness}

Ich habe bei SAPiens teilgenommen, weil...

...durch die Realisierung meiner Idee ein Bedürfnis von mir befriedigt werden kann.

...ich einen Bedarf festgestellt habe, den die SAP-Software bzw. das SAP-Unternehmen nicht erfüllt.

...ich mit der bestehenden SAP-Software bzw. mit den bestehenden Geschäftsstrategien von SAP nicht zufrieden bin.

\section{Involvement}

SAP-Software ist für mich...

unwichtig - wichtig

wenig aufregend - aufregend

langweilig - interessant

\section{Wissen}

Ich verfüge über technisches Wissen im Bereich SAP-Software.

Verglichen mit meinen Freunden kenne ich mich mit der Verwendung von SAPSoftware bzw. dem Unternehmen im Allgemeinen gut aus.

Ich kenne mich mit den Funktionen von SAP-Software gut aus.

\section{Attraktivität als Arbeitgeber}

Ich habe bei SAPiens teilgenommen, weil... ...ich mir erhoffe, durch die Teilnahme später bei der SAP AG zu arbeiten oder eine Abschlussarbeit bei SAP zu schreiben.

...ich SAP von meinen Fähigkeiten überzeugen möchte.

Keywords
balanced scorecard
idea community
Innovation controlling
innovation management
open innovation
virtual community
Summary
Company-based Idea communities on
the internet in which customers can
submit and collaborate on innovation
ideas, are an effective means for inte-
grating customers in open innovation
processes. We present a balanced
scorecard approach for controlling
idea communities. This is grounded in
traditional innovation and virtual com-
munity controlling instruments. We
tested the concept in a real world ldea
Community and show its applicability.

\section{Literatur}

Amabile, T. M., Creativity in context. Update to social psychology of creativity, 1. Aufl., Oxford 1996.

Blohm, I./Bretschneider, U./Leimeister, J. M./ Krcmar, H., Does collaboration among participants lead to better ideas in IT-based idea competitions? An empirical investigation, in: International Journal of Networking and Virtual Organizations, im Erscheinen

Bretschneider, U., Die Ideen Community zur Integration von Kunden in die frühen Phasen des Innovationsprozesses. Empirische Analysen und Implikationen für Forschung und Praxis, Disseration Technische Universität München, Garching b. München 2010.

Brockhoff, K., Forschung und Entwicklung. Planung und Kontrolle, Aufl., München 1999.
Chesbrough, $H$. W., The era of open innovation, in: MIT Sloan Management Review, 44. Jg. (2003), H. 3, S. 35-41.

Cohen, W., M./Levinthal, D., A., Absorptive Capacity: A New Perspective On Learning And Innovation in: Administrative Science Quarterly, 35. Jg. (1990), H. 1, S. 128-152.

Cothrel, J. P., Measuring the success of an online community, in: Strategy \& Leadership, 28. Jg. (2000), H. 2, S. 17-21.

Daum, M., Virtuelle Communities für Krebspatienten, 1. Aufl., Lohmar 2007.

Faber, M. J., Open Innovation. Ansätze, Strategien und Geschäftsmodelle, 1. Aufl., Wiesbaden 2008 .

Hilgers, D./Piller, F. T., Controlling for Open Innovation - Theoretische Grundlagen und praktische Konsequenzen, in: Zeitschrift für Controlling, 21. Jg. (2009), H. 2, S. 5-11.

Janssen, S./Möller, K., Performance Measurement von Produktinnovationen. Konzepte, Instrumente und Kennzahlen des Innovationscontrollings, in: Zeitschrift für Betriebswirtschaft Special Issue, 21. Jg. (2009), H. 2, S. 89-96.

Leimeister, J. M./Krcmar, H., CommunityEngineering: Systematischer Aufbau und Betrieb Virtueller Communitys im Gesundheitswesen, in: Wirtschaftsinformatik, 48. Jg. (2006), H. 6, S. 418-429.

Preece, J., Sociability and usability in online communities: Determining and measuring success in: Behavior and Information Technology Journal 20. Jg. (2001), H. 5, S. 347356

Reichwald, R./Piller, F., Interaktive Wertschöpfung, 2. Aufl., Wiesbaden 2009.

Stieglitz, S., Steuerung Virtueller Communities: Instrumente, Mechanismen, Wirkungszusammenhänge 1. Aufl., Wiesbaden 2008. 